\title{
A MODIFIED CONSERVATION LAW FOR THE PHASE OF THE NONLINEAR SCHRÖDINGER SOLITON
}

\author{
William L. Kath \\ Dedicated to Professor Martin Kruskal, \\ the 'Chuck Yeager' of nonlinear science, \\ on the occasion of his 70th birthday.
}

\begin{abstract}
In the first part of this paper, Noether's theorem and the scale invariance of the nonlinear Schrödinger (NLS) equation are used to derive a modified conservation law for the NLS equation. When used with a soliton solution and applied to the perturbed NLS equation, this modified conservation law is shown to lead naturally to the perturbation equation for a soliton's phase (in a manner similar to the way in which the other, more familiar, conservation laws lead to perturbation equations for a soliton's amplitude, velocity, and position.) Thus, all four of the perturbation equations for the soliton parameters can be obtained directly from conservation laws. These perturbation equations then are applied to an example for which determining phase behavior is important: the parametric amplification of solitons in an optical fiber. The perturbation equations are shown to provide a good approximation to the full perturbed soliton dynamics.
\end{abstract}

\section{Introduction}

The nonlinear Schrödinger (NLS) equation governs pulse propagation in a polarizationpreserving, nonlinear optical fiber when the carrier wavelength is such that anomalous linear group-velocity dispersion is present $[2,14]$. In dimensionless form, the NLS equation is

$$
i \frac{\partial u}{\partial t}+\frac{1}{2} \frac{\partial^{2} u}{\partial x^{2}}+|u|^{2} u=0 .
$$

Here $u$ is the complex-valued envelope of the pulse, $t$ physically represents the normalized spatial variable along the length of the fiber, and $x$ is the normalized reduced time (i.e., shifted to be in a frame of reference which moves with the group velocity of the pulse). We write $x$ and $t$ in the NLS equation (1.1) in the standard mathematical, rather than optical, notational convention [28,31], even though we will discuss the implications of the results for optical applications. (For such optical applications, we can make the replacements $t \rightarrow Z$ and $x \rightarrow T$ to make the physical interpretation more apparent.)

Received March 20, 1996, revised October 9, 1996.

1991 Mathematics Subject Classification: 35Q51, 35Q55, 35B20, 35C20, 78A60.

Key words and phrases: soliton, nonlinear Schrödinger, parametric amplification, perturbation theory, conservation laws, Noether's theorem. 
The NLS equation has the well-known soliton solution

$$
\begin{aligned}
u & =\eta \operatorname{sech} \eta\left(x-x_{0}-V t\right) e^{\frac{1}{2} i\left(\eta^{2}-V^{2}\right) t+i V x+i \varphi} \\
& =\eta \operatorname{sech} \eta\left(x-x_{0}-V t\right) e^{\frac{1}{2} i\left(\eta^{2}+V^{2}\right) t+i V\left(x-x_{0}-V t\right)+i\left(\varphi+V x_{0}\right)}
\end{aligned}
$$

where $\eta, x_{0}, V$, and $\varphi$ are constants. (If the reference frame has been chosen to be centered on the soliton, however, then at least initially $x_{0}=0$ and $V=0$.) There is currently substantial interest in optical solitons due to their potential for application in high-speed optical communication systems [15]. Solitons are ideal candidates as optical 'bits' since they are relatively robust under most types of perturbations, and because solitons with different velocities pass through or interact with one another elastically $[22,32]$.

\section{Noether's theorem, invariances and conservation laws}

Noether's theorem $[11,31]$ states that a conservation law is associated with each invariance of a partial differential equation (or more correctly, with each invariance of the PDE's Lagrangian). Specifically, recall that if one has a functional

$$
J[u]=\int_{\mathcal{R}} F(\vec{x}, u, \vec{\nabla} u) d \vec{x},
$$

and the transformation

$$
\begin{aligned}
v(\vec{y}) & =\Psi(\vec{x}, u, \vec{\nabla} u ; \varepsilon)=u+\varepsilon \psi+\cdots \equiv u+\delta u \\
\vec{y} & =\vec{\Phi}(\vec{x}, u, \vec{\nabla} u ; \varepsilon)=\vec{x}+\varepsilon \vec{\phi}+\cdots \equiv \vec{x}+\overrightarrow{\delta x}
\end{aligned}
$$

yields

$$
J[v]=J[u],
$$

then the functional is invariant under the transformation.

By direct calculation, one finds

$$
\begin{aligned}
J[v]-J[u]= & \int_{\mathcal{R}}\left[F_{u}-\left(F_{u_{x_{i}}}\right)_{x_{i}}\right]\left(\delta u-u_{x_{j}} \delta x_{j}\right) d \vec{x} \\
& +\int_{\mathcal{R}}\left[F_{u_{x_{i}}}\left(\delta u-u_{x_{j}} \delta x_{j}\right)+F \delta x_{i}\right]_{x_{i}} d \vec{x}+\cdots
\end{aligned}
$$

where summation notation is assumed (repeated indices are summed). This result also generalizes to vector dependent variables $\vec{u}$ merely by varying each component separately and then adding the individual results together to get the total variation. The first integral on the right-hand side vanishes since the term in the brackets is the Euler relation associated with the Lagrangian, and that vanishes for a stationary solution, i.e.,

$$
F_{u}-\left(F_{u_{x_{i}}}\right)_{x_{i}}=0 \text {. }
$$

Since $J[v]=J[u]$ and the region $\mathcal{R}$ is in principal arbitrary, we have

$$
\left[F_{u_{x_{i}}}\left(\delta u-u_{x_{j}} \delta x_{j}\right)+F \delta x_{i}\right]_{x_{i}}=0
$$


or equivalently, upon using $\delta u=\varepsilon \psi$ and $\delta \vec{x}=\varepsilon \vec{\phi}$,

$$
\frac{\partial}{\partial x_{i}}\left[F_{u_{x_{i}}}\left(\psi-u_{x_{j}} \phi_{j}\right)+F \phi_{i}\right]=0
$$

To apply this to the NLS equation specifically, one starts with the Lagrangian

$$
L=\frac{1}{2} \int_{-\infty}^{\infty}\left[i\left(u^{*} u_{t}-u u_{t}^{*}\right)-\left|u_{x}\right|^{2}+|u|^{4}\right] d x
$$

and the functional

$$
J[u]=\int L d t=\iint F d x d t .
$$

Here $x_{1}=x$ and $x_{2}=t$, and one varies $u$ and $u^{*}$ independently [27]. Under the transformation $u \rightarrow u+\varepsilon \psi+\cdots, u^{*} \rightarrow u^{*}+\varepsilon \psi^{*}+\cdots, x \rightarrow x+\varepsilon \phi+\cdots$, and $t \rightarrow t+\varepsilon \theta+\cdots,(2.8)$ becomes

$$
\begin{aligned}
& \frac{\partial}{\partial t}\left[F_{u_{t}}\left(\psi-u_{x} \phi-u_{t} \theta\right)+F_{u_{t}^{*}}\left(\psi^{*}-u_{x}^{*} \phi-u_{t}^{*} \theta\right)+F \theta\right] \\
& \quad+\frac{\partial}{\partial x}\left[F_{u_{x}}\left(\psi-u_{x} \phi-u_{t} \theta\right)+F_{u_{x}^{*}}\left(\psi^{*}-u_{x}^{*} \phi-u_{t}^{*} \theta\right)+F \phi\right]=0 .
\end{aligned}
$$

In addition, when integrated with respect to $x$, this becomes

$$
\frac{d}{d t} \int_{-\infty}^{\infty}\left[F_{u_{t}}\left(\psi-u_{x} \phi-u_{t} \theta\right)+F_{u_{t}^{*}}\left(\psi^{*}-u_{x}^{*} \phi-u_{t}^{*} \theta\right)+F \theta\right] d x=0,
$$

which is one of the standard forms for a conservation law $[1,31]$.

The various invariances of the NLS equation now can be used to generate the corresponding conservation laws. The first invariance is with respect to phase: the transformation $u \rightarrow e^{i \varepsilon} u$ (and $u^{*} \rightarrow e^{-i \varepsilon} u^{*}$ ) leaves both the NLS equation and the Lagrangian unchanged. For small $\varepsilon$, this gives $\psi=i u$ (and $\psi^{*}=-i u^{*}$ ), $\phi=0$, and $\theta=0$, which yields

$$
\frac{d}{d t} \int_{-\infty}^{\infty}|u|^{2} d x=0
$$

Next, the NLS equation is invariant with respect to translations in space and time. The former means invariance when $x \rightarrow x-x_{0}$, in which case $\psi=0, \theta=0$, and $\phi=1$, giving

$$
\frac{d}{d t} \int_{-\infty}^{\infty} \frac{i}{2}\left(u^{*} u_{x}-u u_{x}^{*}\right) d x=0
$$

the latter means invariance when $t \rightarrow t-t_{0}$, whence $\psi=0, \phi=0$, and $\theta=1$, giving

$$
\frac{d}{d t} \int_{-\infty}^{\infty}\left(\left|u_{x}\right|^{2}-|u|^{4}\right) d x=0
$$

These conservation laws are identified with conservation of mass (or photon number, in the case of optical solitons), momentum, and energy [19,21]. When used for soliton perturbation theory (as described in Section 3), however, these conservation laws give information about only two of the soliton parameters, namely the soliton amplitude $\eta$ and frequency shift or velocity $V$; no information about the other parameters $x_{0}$ and $\varphi$ is provided, since each of the above conservation laws is phase and translation invariant. Note that the result obtained from the energy conservation law (2.15) for 
$\eta$ and $V$ is consistent with the results obtained from the other two conservation laws, so no additional information is obtained from it.

The NLS equation and its Lagrangian are also Galilean invariant, however, which means that if $u(x, t)$ is a solution, then so is $u(\hat{x}-\varepsilon \hat{t}, \hat{t}) e^{i \varepsilon \hat{x}-i \varepsilon^{2} \hat{t} / 2}$. This is equivalent to the transformation

$$
\hat{x}=x+\varepsilon t, \quad \hat{t}=t, \quad \hat{u}=e^{i \varepsilon \hat{x}-i \varepsilon^{2} \hat{t} / 2} u .
$$

Thus, $\psi=i x u, \phi=t$, and $\theta=0$, giving

$$
\frac{d}{d t} \int_{-\infty}^{\infty}\left[x|u|^{2}+t \frac{i}{2}\left(u^{*} u_{x}-u u_{x}^{*}\right)\right] d x=0
$$

or equivalently,

$$
\frac{d}{d t} \int_{-\infty}^{\infty} x|u|^{2} d x=\int_{-\infty}^{\infty} \frac{i}{2}\left(u u_{x}^{*}-u^{*} u_{x}\right) d x
$$

Note that this is not the standard form for a conservation law, in that the right-handside is non-zero. As a result, it will be called a modified conservation law because of the similarity with the definition of a modified (or generalized) eigenvector or eigenfunction [10]. It will be shown shortly that this modified conservation law gives an equation for the soliton position $x_{0}$ (i.e., the center of mass).

The previous three conservation laws for the NLS equation are well-known [12]. The NLS equation has one more conservation law, however, one that comes about due to scale invariance. The NLS is scale invariant because if $u(x, t)$ is a solution, then so is

$$
a u\left(\frac{x}{a}, \frac{t}{a^{2}}\right)
$$

The Lagrangian is not scale invariant, however. Under the transformation

$$
u(x, t) \rightarrow a u\left(\frac{x}{a}, \frac{t}{a^{2}}\right)
$$

we have

$$
J[u] \rightarrow a J[u]
$$

Noether's theorem does not directly apply to this situation, but it can be easily modified. Since $J[v]=a J[u]$, equivalently,

$$
J[v]=J[u]+(a-1) J[u] .
$$

In addition to the terms in (2.11) resulting from the changes in $u, x$, and $t$, there is now an extra term from the term proportional to $(a-1)$ on the right-hand side of (2.22). If $a=1+\varepsilon,(2.11)$ then becomes

$$
\begin{aligned}
& \frac{\partial}{\partial t}\left[F_{u_{t}}\left(\psi-u_{x} \phi-u_{t} \theta\right)+F_{u_{t}^{*}}\left(\psi^{*}-u_{x}^{*} \phi-u_{t}^{*} \theta\right)+F \theta\right] \\
& \quad+\frac{\partial}{\partial x}\left[F_{u_{x}}\left(\psi-u_{x} \phi-u_{t} \theta\right)+F_{u_{x}^{*}}\left(\psi^{*}-u_{x}^{*} \phi-u_{t}^{*} \theta\right)+F \phi\right]=F,
\end{aligned}
$$

where $\psi=u, \phi=-x$, and $\theta=-2 t$. Equivalently, in integrated form this is

$$
\frac{d}{d t} \int_{-\infty}^{\infty}\left[F_{u_{t}}\left(\psi-u_{x} \phi-u_{t} \theta\right)+F_{u_{t}^{*}}\left(\psi^{*}-u_{x}^{*} \phi-u_{t}^{*} \theta\right)+F \theta\right] d x=\int_{-\infty}^{\infty} F d x
$$


and (2.24) thus gives the additional modified conservation law

$$
\frac{i}{2} \int_{-\infty}^{\infty}\left(u u_{t}^{*}-u^{*} u_{t}\right) d x-\frac{d}{d t} \int_{-\infty}^{\infty} \frac{i}{2} x\left(u u_{x}^{*}-u^{*} u_{x}\right) d x=-\frac{3}{2} \int_{-\infty}^{\infty}\left(\left|u_{x}\right|^{2}-|u|^{4}\right) d x
$$

It will be shown in the next section that this modified conservation law (which does not appear to be widely known) provides an equation for the soliton phase $\varphi$.

Note that when the solution $u$ is symmetric about $x=0$, the second term on the left-hand side of the above equation vanishes, and (2.25) can be interpreted as the result of multiplying the NLS equation by $u^{*}$, taking the imaginary part, and integrating. This basic procedure was used by Fauve and Thual [9]; however, they did not point out the more general form or its connection with the scale invariance of the NLS equation.

\section{Soliton perturbation theory using the conservation laws}

The conservation laws (2.13) and (2.14) and modified conservation laws (2.18) and (2.25) form the basis for a perturbation theory analysis of the four soliton parameters $\left(\eta, V, x_{0}\right.$, and $\left.\varphi\right)$. Such an analysis is relevant when additional terms, represented by $f(x, t)$, appear in the NLS equation to give

$$
\frac{\partial u}{\partial t}=\frac{i}{2} \frac{\partial^{2} u}{\partial x^{2}}+i|u|^{2} u+f
$$

In this case, by direct calculation, the conservation laws are modified and become

$$
\begin{gathered}
\frac{d}{d t} \int_{-\infty}^{\infty}|u|^{2} d x=2 \Re \int_{-\infty}^{\infty} u^{*} f d x \\
\frac{d}{d t} \int_{-\infty}^{\infty} \frac{i}{2}\left(u u_{x}^{*}-u^{*} u_{x}\right) d x=-2 \Im \int_{-\infty}^{\infty} u_{x}^{*} f d x \\
\frac{d}{d t} \int_{-\infty}^{\infty} x|u|^{2} d x=\int_{-\infty}^{\infty} \frac{i}{2}\left(u u_{x}^{*}-u^{*} u_{x}\right) d x+2 \Re \int_{-\infty}^{\infty} x u^{*} f d x, \quad \text { and } \\
\frac{i}{2} \int_{-\infty}^{\infty}\left(u u_{t}^{*}-u^{*} u_{t}\right) d x-\frac{d}{d t} \int_{-\infty}^{\infty} \frac{i}{2} x\left(u u_{x}^{*}-u^{*} u_{x}\right) d x= \\
-\frac{3}{2} \int_{-\infty}^{\infty}\left(\left|u_{x}\right|^{2}-|u|^{4}\right) d x+2 \Im \int_{-\infty}^{\infty}\left(u^{*}+x u_{x}^{*}\right) f d x
\end{gathered}
$$

The additional term $f(x, t)$ produces two kinds of effects on the solution $u$ : it perturbs the soliton parameters, and it also generates a small amount of dispersive radiation. To first order, these two effects do not interact with one another, and therefore the changes in the soliton parameters can be evaluated by substituting the soliton shape (1.2) into the various conservation laws [19]. (In some cases, the effect of the dispersive radiation can be evaluated with an associated field $[7,12])$. Rather than (1.2), however, it is a bit more convenient to use the slightly different soliton solution

$$
u=\eta \operatorname{sech} \eta(x-\xi) e^{i \kappa(x-\xi)+i \vartheta}=\eta \operatorname{sech} \zeta e^{i \sigma}
$$

where, for convenience, we have defined

$$
\zeta=\eta(x-\xi)
$$


and

$$
\sigma=\kappa(x-\xi)+\vartheta=\frac{\kappa}{\eta} \zeta+\vartheta
$$

When this is substituted into the conservation laws (3.2)-(3.5), one obtains the following equations for the soliton parameters:

$$
\begin{aligned}
\frac{d \eta}{d t} & =\Re \int_{-\infty}^{\infty} \operatorname{sech} \zeta e^{-i \sigma} f d \zeta \\
\frac{d \kappa}{d t} & =\Im \int_{-\infty}^{\infty} \operatorname{sech} \zeta \tanh \zeta e^{-i \sigma} f d \zeta \\
\frac{d \xi}{d t} & =\kappa+\frac{1}{\eta^{2}} \Re \int_{-\infty}^{\infty} \zeta \operatorname{sech} \zeta e^{-i \sigma} f d \zeta \\
\frac{d \vartheta}{d t} & =\kappa \frac{d \xi}{d t}+\frac{1}{2}\left(\eta^{2}-\kappa^{2}\right)+\frac{1}{\eta} \Im \int_{-\infty}^{\infty}(1-\zeta \tanh \zeta) \operatorname{sech} \zeta e^{-i \sigma} f d \zeta
\end{aligned}
$$

These perturbation equations are identical with those obtained by using the inverse scattering transform $[1,17,19,25,28]$.

An alternative interpretation of the above soliton perturbation theory is as a special case of collective coordinates [13]. Essentially, when one substitutes a trial function with arbitrary time-dependent parameters in the Lagrangian, one obtains ordinary differential equations for those parameters [4-6]. The form of the trial function used in the Lagrangian can be more general than that of the soliton solution [29] (sometimes a more general form gives a better approximation [30]), but when the soliton solution is used as a trial function, (3.9)-(3.12) are obtained. Since it has been shown here that all of the soliton perturbation equations arise from conservation laws associated with invariances of the NLS Lagrangian, it is not surprising that a direct derivation of ODEs from the same Lagrangian using the collective coordinate approach yield exactly the same equations.

\section{An application to soliton control using phase-sensitive amplification}

One application where the phase evolution is particularly important is the control of optical solitons using phase-sensitive parametric amplification $[23,24,26]$. (A mathematically similar fluid-mechanical problem is the parametric driving of surface waves $[8,20]$.) In the case of optical solitons, the perturbing term $f$ in (3.1) is

$$
f=-\gamma u+g e^{i \nu t} u^{*}
$$

where $\gamma$ is the linear loss in the fiber, $g$ is parametric gain from the amplifier, and $\nu$ is a detuning parameter (i.e., the rate of phase advance of the amplifier relative to the NLS carrier). The phase-sensitive amplification usually arises from discrete, periodically-spaced (or lumped) amplifiers. The above equation arises when the loss and amplification are relatively weak $[8,26]$. When the loss and gain are strong, a different evolution equation is obtained $[23,24]$.

In general the parametric gain $g$ can vary, as is the case when the amplifier is driven by a modulated or mode-locked pump laser. Here this will be modeled by assuming

$$
g=g_{0}(1+\beta \cos \omega x) \text {. }
$$


(Recall that in the optical fiber application being considered here, $x$ represents the normalized reduced time, measured across the soliton profile, while $t$ represents distance along the fiber.) When the perturbation equations (3.9)-(3.12) are used, one obtains

$$
\begin{aligned}
& \frac{d \eta}{d t}=-2 \gamma \eta+2 g_{0} \eta F_{1}\left(\frac{2 \kappa}{\eta}\right) \cos 2 \Phi \\
& +g_{0} \beta \eta\left[F_{1}\left(\frac{\omega-2 \kappa}{\eta}\right) \cos (\omega \xi-2 \Phi)+F_{1}\left(\frac{\omega+2 \kappa}{\eta}\right) \cos (\omega \xi+2 \Phi)\right] \text {, } \\
& \frac{d \kappa}{d t}=-g_{0} \eta F_{2}\left(\frac{2 \kappa}{\eta}\right) \cos 2 \Phi \\
& +\frac{g_{0} \beta \eta}{2}\left[F_{2}\left(\frac{\omega-2 \kappa}{\eta}\right) \cos (\omega \xi-2 \Phi)-F_{2}\left(\frac{\omega+2 \kappa}{\eta}\right) \cos (\omega \xi+2 \Phi)\right] \\
& \frac{d \xi}{d t}=\kappa-\frac{g_{0}}{\eta} F_{3}\left(\frac{2 \kappa}{\eta}\right) \sin 2 \Phi \\
& -\frac{g_{0} \beta}{2 \eta}\left[F_{3}\left(\frac{\omega-2 \kappa}{\eta}\right) \sin (\omega \xi-2 \Phi)+F_{3}\left(\frac{\omega+2 \kappa}{\eta}\right) \sin (\omega \xi+2 \Phi)\right]
\end{aligned}
$$

and

$$
\begin{aligned}
\frac{d \Phi}{d t}=\kappa & \frac{d \xi}{d t}+\frac{1}{2}\left(\eta^{2}-\kappa^{2}\right)-\frac{\nu}{2}-g_{0} F_{4}\left(\frac{2 \kappa}{\eta}\right) \sin 2 \Phi \\
& +\frac{g_{0} \beta}{2}\left[F_{4}\left(\frac{\omega-2 \kappa}{\eta}\right) \sin (\omega \xi-2 \Phi)-F_{4}\left(\frac{\omega+2 \kappa}{\eta}\right)\right] \sin (\omega \xi+2 \Phi)
\end{aligned}
$$

Here $2 \Phi=2 \vartheta-\nu t$ and

$$
\begin{aligned}
& F_{1}(k)=k \pi / \sinh (k \pi / 2) \equiv 2 F(k), \\
& F_{2}(k)=k F(k), \\
& F_{3}(k)=-2 F^{\prime}(k), \\
& F_{4}(k)=F(k)-k F^{\prime}(k) .
\end{aligned}
$$

It also is useful to consider the above equations when $\kappa$ is small since they simplify considerably:

$$
\begin{aligned}
\frac{d \eta}{d t} & \approx-2 \gamma \eta+2 g_{0} \eta \cos 2 \Phi+2 g_{0} \beta \eta F_{1}(\omega / \eta) \cos (\omega \xi) \cos 2 \Phi \\
\frac{d \kappa}{d t} & \approx-2 g_{0} \kappa \cos 2 \Phi+g_{0} \beta \eta F_{2}(\omega / \eta) \sin (\omega \xi) \sin 2 \Phi \\
\frac{d \xi}{d t} & \approx \kappa-\frac{\pi^{2} g_{0}}{3 \eta^{2}} \kappa \sin 2 \Phi-\frac{g_{0} \beta}{\eta} F_{3}(\omega / \eta) \sin (\omega \xi) \cos 2 \Phi, \quad \text { and } \\
\frac{d \Phi}{d t} & \approx \frac{1}{2} \eta^{2}-\frac{\nu}{2}-g_{0} \sin 2 \Phi-g_{0} \beta F_{4}(\omega / \eta) \cos (\omega \xi) \sin 2 \Phi
\end{aligned}
$$


When $\beta=0$ (the case of no gain modulation), it is easy to see that a steady state occurs when $\kappa=0$ and

$$
\begin{aligned}
\cos 2 \Phi & =\gamma / g_{0}, \\
\eta^{2} & =\nu+2 \gamma \tan 2 \Phi .
\end{aligned}
$$

Note this reproduces the exact solution of the parametrically-driven NLS equation $[8,26]$. The steady-state value of $\xi$ is arbitrary; this is to be expected since when $\beta=0$, the equation is translation invariant. (But if $\kappa=0$ initially then it remains zero, and $\xi$ retains its initial value.) Two solutions therefore are found (corresponding to the two possible values of $\Phi$; the sign of $\eta$ is ignored); the larger of these solutions is stable while the smaller is unstable $[8,9]$.

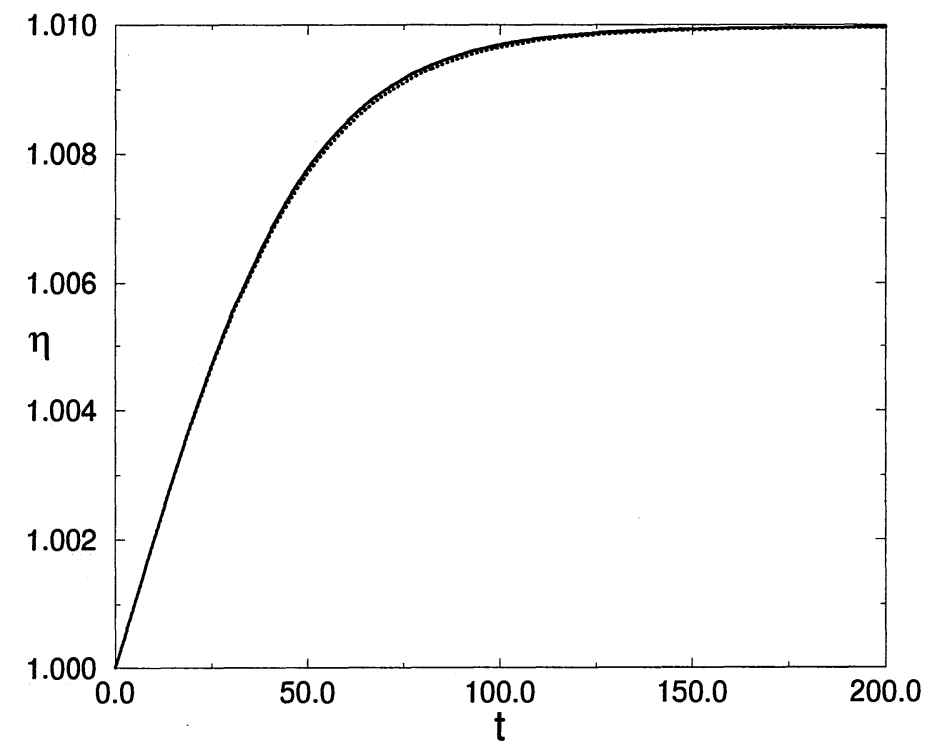

FIGURE 1. A comparison of the solution of the NLS equation plus parametric driving (solid line) with the solution of the perturbation equations, (4.3)-(4.6) (dotted line) in the case of no gain modulation $(\beta=0)$. The curves plotted are for the time evolution of the soliton amplitude $\eta$; in the case of the full NLS solution $\eta$ was determined from its integral definition, $\frac{1}{2} \int|u|^{2} d x$. Here $\gamma=0.5$ and $g_{0}=0.5001$. The initial values of $\eta, \kappa$, and $\Phi$ were $1.0,0.0$, and 0.0 , respectively.

In Figure 1, we compare the solution of the parametrically-driven NLS equation, (3.1) with perturbation $f$ given by (4.1), with the solution of the perturbation equations, (4.3)-(4.6). Note that for these particular values of $\gamma$ and $g_{0}$, the agreement between the two solutions is very good. For these parameter values, the oscillation is overdamped. Figure 2 gives another example with different values of $\gamma$ and $g_{0}$, which also shows excellent agreement. For these parameters, a damped oscillation results.

The best agreement between the solution of the approximate equations (4.3)-(4.6) and the solution of the perturbed NLS equation is found when the difference between $\gamma$ and $g_{0}$ is small, so that the steady-state value of the angle $\Phi$ is small. In particular, it 


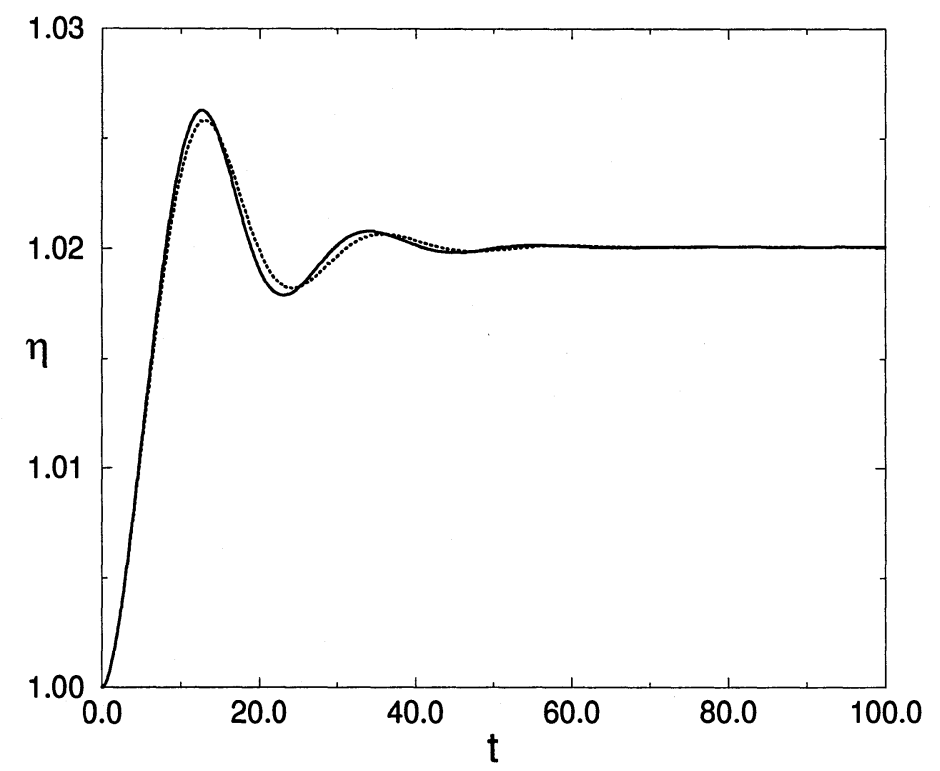

FIGURE 2. A second example of the comparison between the solution of the NLS equation plus parametric driving (solid line) with the solution of the perturbation equations, (4.3)-(4.6) (dotted line) in the case of no gain modulation. Here $\gamma=0.1, g_{0}=0.102034$, and the initial value of $\Phi$ was 0.1 .

appears that for good agreement, the steady-state value of $\Phi$ should be small compared with $\gamma$ and $g_{0}$. The cases represented in Figures 1 and 2 both satisfy this condition. In Figure 3, a case where the steady-state value of $\Phi$ is not small in comparison with $\gamma$ and $g_{0}$ is shown. Now the approximate equations give good agreement for short and long times, but the transient is not properly resolved.

In Figure 3, the parameter $\eta$ is calculated from the full NLS solution in two different ways, both by integrating $|u|^{2}$ and from the maximum of $|u|$. If the solution has a hyperbolic secant shape, then these two results will be the same. Since they do not agree in Figure 3, this means that during the transient evolution the pulse shape deviates from the fixed hyperbolic secant shape assumed in deriving the approximate equations. These shape deformations can be interpreted as resulting from the secondorder coupling between the discrete eigenmodes associated with the soliton and the continuous spectrum [18].

A linearized analysis about the steady-state solution [26] suggests that the linearized eigenfunctions and eigenvalues are significantly perturbed when $\Phi$ is not small in comparison with $\gamma$ and $g_{0}$. This also is consistent with Figure 3, since the decay rates found in the full NLS solution and the approximate equations are different (which in essence use the unperturbed eigenfunctions and eigenvalues since the approximate equations assume an unperturbed hyperbolic secant shape). Determining these perturbed eigenfunctions and eigenvalues (in general, this must be done numerically) should shed some light upon the pulse reshaping that is occurring. Alternatively, it is possible to use variational methods (i.e., more general collective coordinates) or 


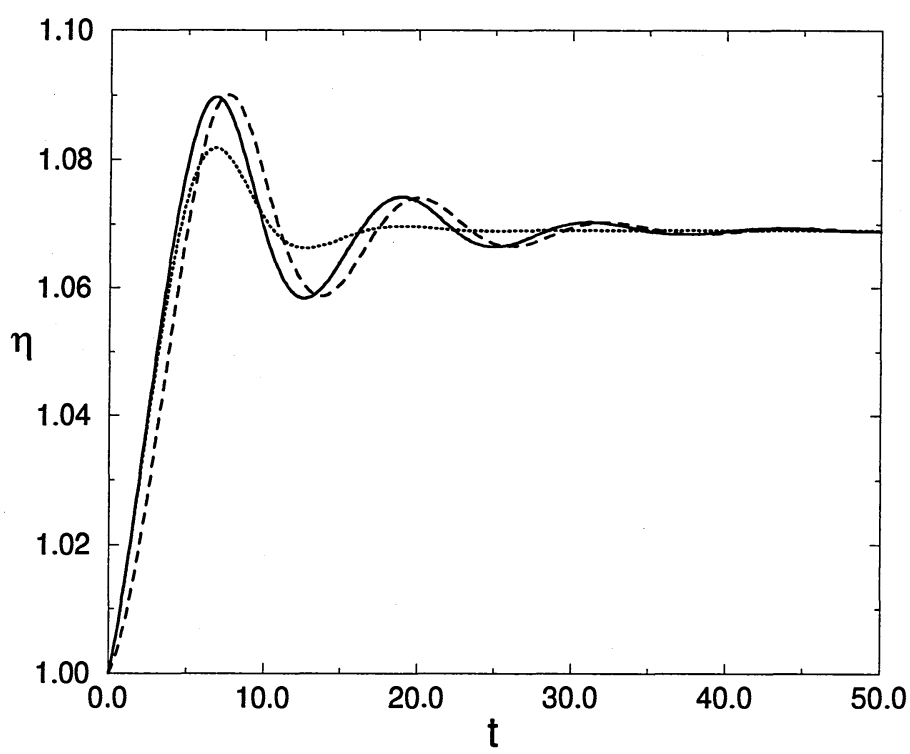

FIGURE 3. A third example of the comparison between the solution of the NLS equation plus parametric driving (solid and dashed lines) with the solution of the perturbation equations, (4.3)-(4.6) (dotted line) in the case of no gain modulation. The value of $\eta$ in the full solution was determined both from its integral definition (solid line) and from the maximum of $|u|$ (dashed line). Here $\gamma=0.25, g_{0}=0.26$, and the initial value of $\Phi$ was 0.1 .

moments of the conservation laws to incorporate some shape changes into the approximate evolution equations $[3,16,29]$. These additional studies are currently in progress.

When gain modulation is present $(\beta>0)$, the perturbed NLS equation is no longer translationally invariant, and obtaining steady-state solutions now also requires that $\omega \xi=n \pi$ (at least when the perturbation is small). The perturbation equations (4.3)(4.6) predict that when the difference between $g_{0}$ and $\gamma$ is small compared with $g_{0}$ (so that $\Phi$ is small) the even $n$ solutions are stable, while the odd $n$ solutions are unstable. (The precise expression is somewhat complicated to write down, but can be obtained in a straightforward manner from (4.3)-(4.6).) This means that a perturbed soliton becomes locked to the pattern produced by the modulated gain, and in particular the pulses move to positions of maximum gain.

Figure 4 shows a comparison of the position $\xi$ calculated from both the solution of parametrically-driven NLS equation and the solution of the perturbation equations, (4.3)-(4.6). The two solutions are in good agreement, and for these parameters the pulse position $\xi=0$ is stable. Figure 5 compares the results for the pulse frequency shift, $\kappa$, and Figure 6 compares the results for the pulse amplitude, $\eta$.

Note that there is now some discrepancy between the two calculated values of the steady-state value of $\eta$. This appears to be due to pulse shaping (or deformation) caused by the modulated gain. As mentioned previously, since the perturbation solution fixes the shape of the pulse to be a hyperbolic secant, no such adjustment of 


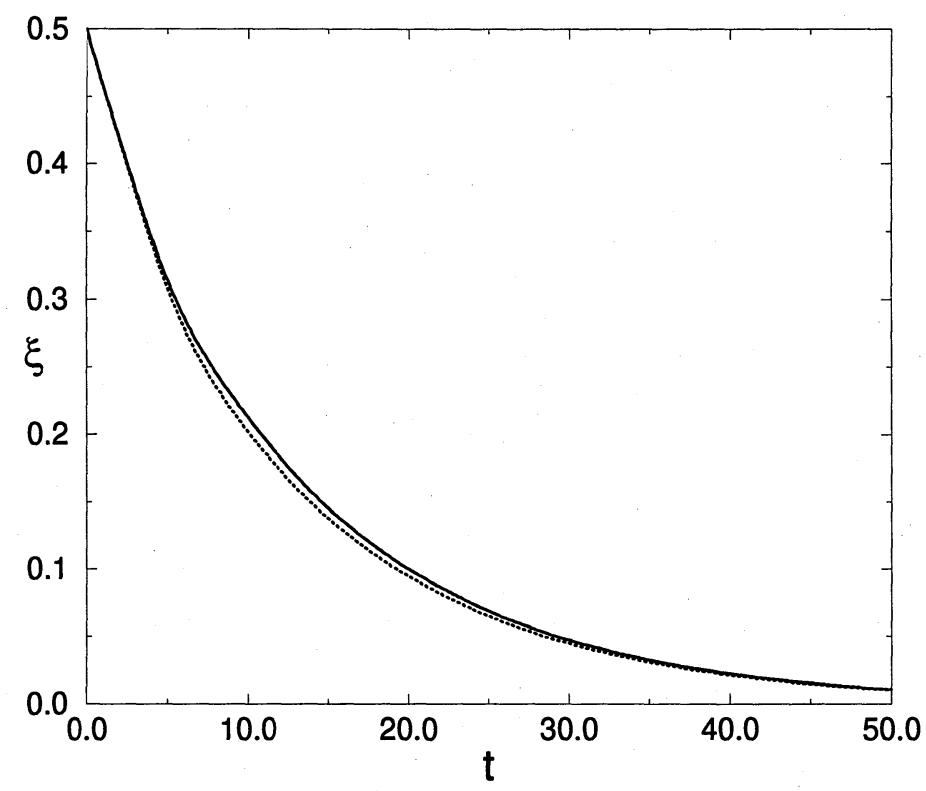

FIGURE 4. Comparison of the solution of pulse position $\xi$ calculated from the NLS equation plus parametric driving (solid line) with the solution calculated from the perturbation equations, (4.3)-(4.6), (dotted line). The two solutions are in good agreement, and both show that the position $\xi=0$ is stable. Here $\gamma=0.5, g_{0}=0.5, \beta=0.2$, and $\omega=2.0$. The initial values of $\eta, \kappa, \xi$, and $\Phi$ were $1,0,0.5$, and 0.25 , respectively.

shape is possible in the approximate solution. Such pulse reshaping is expected when modulated gain is used, particularly when the modulation period (here $2 \pi / \omega=\pi$ ) is comparable to the pulse width. This reshaping can be seen directly if one compares the two final steady-states, as shown in Figure 7. Here the smaller gain in the vicinity of $x= \pm \pi$ causes the tails of the pulse to be reduced relative to the ideal hyperbolic secant shape. Nevertheless, even with this pulse reshaping the predictions the approximate equations make for the pulse position $\xi$ and frequency shift $\kappa$ are quite good.

\section{Conclusions}

Noether's theorem and the scale invariance of the nonlinear Schrödinger (NLS) equation have been used to derive a modified conservation law for the NLS equation, and this conservation was shown to lead naturally to the perturbation equation for a soliton's phase which previously has only been obtained via inverse scattering theory. When combined with three other known conservation laws, the result is that all four perturbation equations for a soliton's parameters (amplitude, phase, position, and frequency shift) can be obtained directly from conservation laws. 


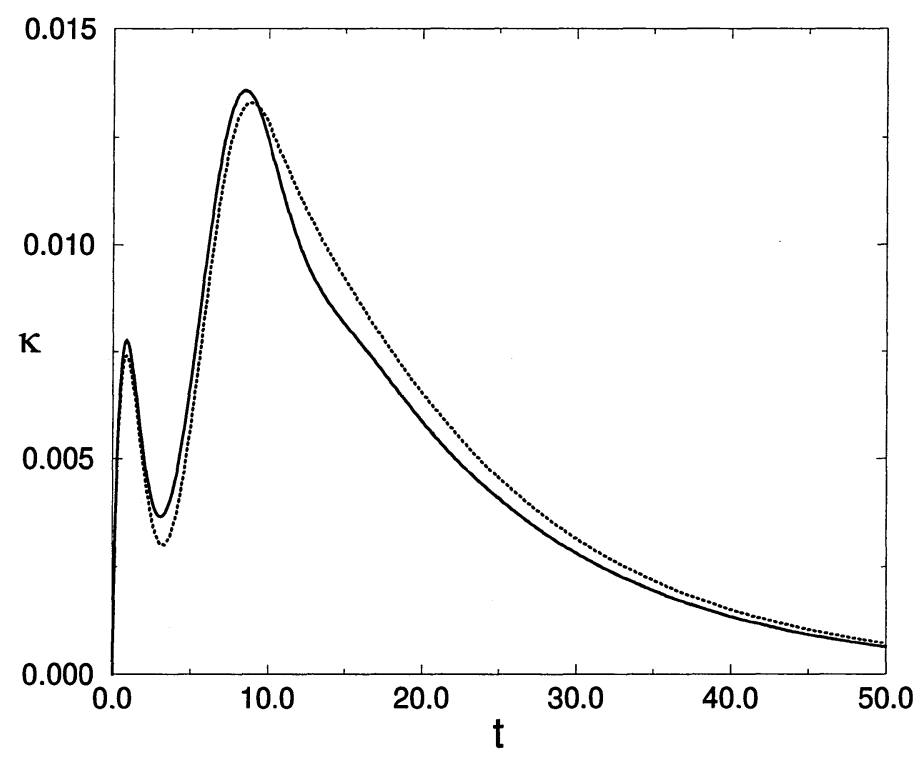

FIgURE 5. Comparison of the solution of pulse frequency shift $\kappa$ calculated from the NLS equation plus parametric driving (solid line) with the solution calculated from the perturbation equations, Eqs. (4.3)-(4.6), (dotted line). The parameters are the same as in Figure 4.

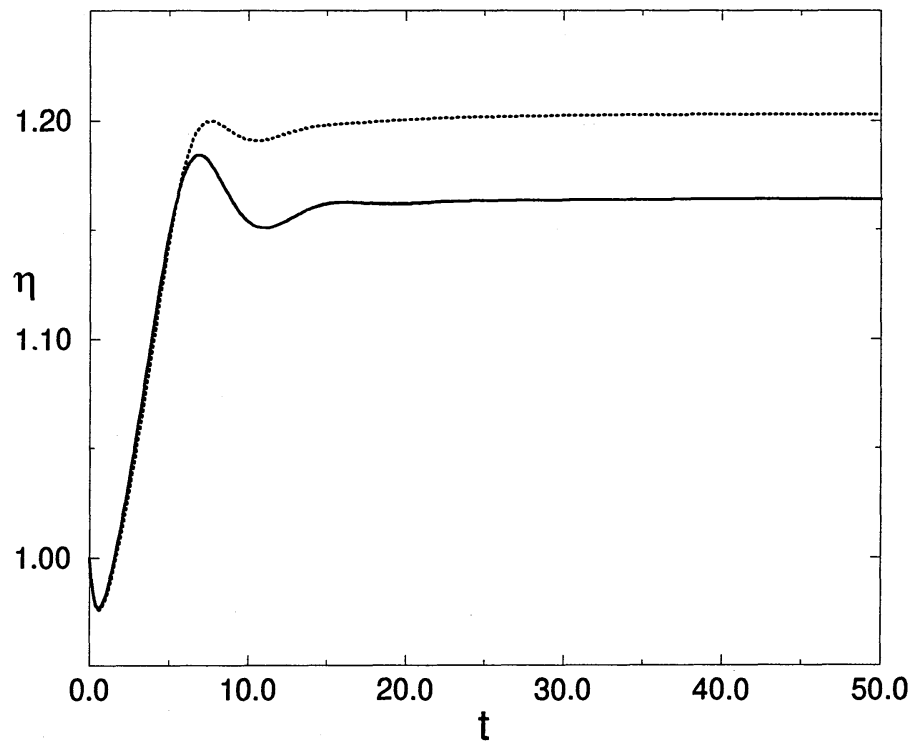

FIgURE 6. Comparison of the solution of pulse amplitude $\eta$ calculated from the NLS equation plus parametric driving (solid line) with the solution calculated from the perturbation equations, Eqs. (4.3)(4.6), (dotted line). The parameters are the same as in Figure 4. 


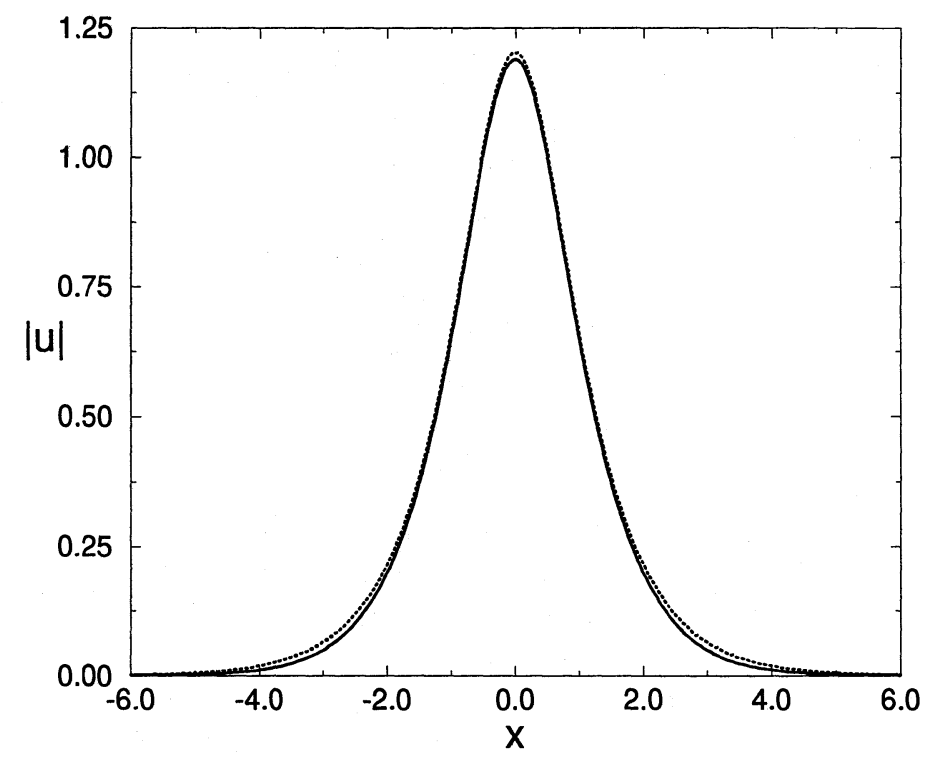

FiguRE 7. Comparison of the final steady-state solution profiles obtained from the NLS equation plus parametric driving (solid line) and from the perturbation equations, Eqs. (4.3)-(4.6), (dotted line), showing a slight amount of pulse reshaping. The parameters are the same as in Figure 4.

In addition, the perturbation equations have been applied to an example for which determining phase behavior is important, namely the parametric amplification of solitons in an optical fiber. The perturbation equations were shown to provide a good approximation to the full perturbed soliton dynamics in a number of cases. Of particular interest is the case when gain modulation is present, since in this case the perturbed NLS equation is no longer translationally invariant, and a soliton locks stably to one of the maxima of the gain profile. This behavior has been proposed as a method for stabilizing solitons stored in an optical fiber memory loop against timing fluctuations [26].

One potential drawback of the soliton perturbation theory is that pulse reshaping or deformation is not included in the approximations that are made. Such reshaping is clearly present when the NLS equation is parametrically perturbed. As a result, careful comparisons need to be made between the approximate equations and full numerical solutions in order to determine the range of parameter values over which the perturbation equations give reasonable results. In addition, further work is necessary to explore possible methods for including the effects of such pulse reshaping in the approximate evolution equations.

Acknowledgements. This work was supported in part by the National Science Foundation under grant number DMS 95-00615 and by the Air Force Office of Scientific Research, Air Force Materials Command, USAF under grant number 93-1-0084. 
Disclaimer. The US Government is authorized to reproduce and distribute reprints for governmental purposes notwithstanding any copyright notation thereon. The views and conclusions contained herein are those of the authors and should not be interpreted as necessarily representing the official policies or endorsements, either expressed or implied, of the Air Force Office of Scientific Research or the US Government.

\section{References}

1. M. J. Ablowitz and H. Segur, Solitons and the Inverse Scattering Transform, Society for Industrial and Applied Mathematics, Philadelphia, 1981.

2. G. P. Agrawal, Nonlinear Fiber Optics Academic Press, San Diego, second edition, 1995.

3. D. Anderson, Variational approach to nonlinear pulse propagation in optical fibers, Phys. Rev. A 27 (1983), 3135-3145.

4. D. Anderson, M. Lisak, and T. Reichel, Asymptotic propagation properties of pulses in a solitonbased optical-fiber communication system, J. Opt. Soc. Amer. B 5 (1995), 1166-1174.

5. A. Bondeson, M. Lisak, and D. Anderson, Soliton perturbations: a variational principle for the soliton parameters, Phys. Scr. 20 (1979), 479-485.

6. J. G. Caputo, N. Flytzanis, and M. P. Sørensen, Ring laser configuration studied by collective coordinates, J. Opt. Soc. Amer. B 12 (1995), pp. 139-145.

7. J. N. Elgin, Perturbations of optical solitons, Phys. Rev. A 47 (1993), 4331-4341.

8. C. Elphick and E. Meron, Localized structures in surface waves, Phys. Rev. A 40 (1989), 32263229.

9. S. Fauve and O. Thual, Solitary waves generated by subcritical instabilities in dissipative systems, Phys. Rev. Lett. 64 (1990), 282-284.

10. B. Friedman, Principles and Techniques of Applied Mathematics, Dover, New York, 1990.

11. I. M. Gelfand and S. V. Fomin, Calculus of Variations, Prentice-Hall, Englewood Cliffs, New Jersey, 1963.

12. J. P. Gordon, Dispersive perturbations of solitons of the nonlinear Schrödinger equation, J. Opt. Soc. Amer. B 9 (1992), 91-97.

13. V. Guillemin and S. Sternberg, Symplectic Techniques in Physics, Cambridge University Press, Cambridge, England, 1984.

14. A. Hasegawa and Y. Kodama, Solitons in Optical Communications, Oxford University Press, Oxford, 1995.

15. H. A. Haus, Molding light into solitons, IEEE Spectrum (March 1993), 48-53.

16. W. L. Kath and N. F. Smyth, Soliton evolution and radiation loss for the nonlinear Schrödinger equation, Phys. Rev. E 51 (1995), 1484-1492.

17. D. J. Kaup, Perturbation theory for solitons in optical fibers, Phys. Rev. A 42 (1990), 5689-5694.

18. _ Second-order perturbations for solitons in optical fibers, Phys. Rev. A 44 (1991), 45824590.

19. D. J. Kaup and A. C. Newell, Solitons as particles, oscillators, and in slowly changing media: A singular perturbation theory, Proc. R. Soc. Lond. A 361 (1978), 413-446.

20. J. R. Keolian Wu and I. Rudnick, Observation of a nonpropagating hydrodynamic soliton, Phys. Rev. Lett. 52 (1984), 1421-1424.

21. A. M. Kosevich, Particle and wave properties of solitons, Physica D 41 (1990), 253-261.

22. M. D. Kruskal, The Korteweg-de Vries equation and related evolution equations. In: Nonlinear Wave Motion, Lectures in Applied Mathematics 15 (Ed., A. C. Newell), American Mathematical Society, Providence, RI (1974), pp.61-83.

23. J. N. Kutz, C. V. Hile, W. L. Kath, R.-D. Li, and P. Kumar, Pulse propagation in nonlinear optical fiber-lines that employ phase-sensitive parametric amplifiers, J. Opt. Soc. Amer. B 11 (1994), 2112-2123.

24. J. N. Kutz and W. L. Kath, Stability of pulses in nonlinear optical fibers using phase-sensitive amplifiers, SIAM J. Appl. Math. 56 (1996), 611-626.

25. G. L. Lamb, Jr, Elements of Soliton Theory, Wiley, New York, 1980.

26. A. Mecozzi, W. L. Kath, P. Kumar, and C. G. Goedde, Long-term storage of a soliton bit stream by use of phase-sensitive amplification, Opt. Lett. 19 (1994), 2050-2052. 
27. P. M. Morse and H. Feshbach, Methods of Theoretical Physics, McGraw-Hill, New York, 1953.

28. A. C. Newell, Solitons in Mathematics and Physics, Society for Industrial and Applied Mathematics, Philadelphia, 1985.

29. T. Ueda and W. L. Kath, Dynamics of coupled solitons in nonlinear optical fibers, Phys. Rev. A 42 (1990), 563-571.

30. Q. Wang, P. K. A. Wai, C.-J. Chen, and C. R. Menyuk, Numerical modeling of soliton-dragging logic gates, J. Opt. Soc. Amer. B 10 (1993), 2030-2039.

31. G. B. Whitham, Linear and Nonlinear Waves, Wiley, New York, 1974.

32. N. J. Zabusky and M. D. Kruskal, Interaction of "solitons" in a collisionless plasma and the recurrence of initial states, Phys. Rev. Lett. 15 (1965), 240-243.

Engineering Sciences and Applied Mathematics, McCormick School of Engineering ANd Applied Science, NorthWestern University, Evanston, Illinois 60208-3125 